Service social

\title{
Vers le développement de l'autonomie économique des femmes : une expérience de groupe
}

\section{Pascaline Cosse}

Volume 42, numéro 2, 1993

Recherche et pensée critique

URI : https://id.erudit.org/iderudit/706623ar

DOI : https://doi.org/10.7202/706623ar

Aller au sommaire du numéro

Éditeur(s)

École de service social de l'Université Laval

ISSN

1708-1734 (numérique)

Découvrir la revue

Citer cette note

Cosse, P. (1993). Vers le développement de l'autonomie économique des femmes : une expérience de groupe. Service social, 42(2), 163-182. https://doi.org/10.7202/706623ar
Résumé de l'article

Les femmes, en particulier celles issues de milieux populaires, vivent souvent dans des conditions d'impuissance et d'oppression sur le plan socio-économique. Après un aperçu des facteurs qui les placent dans cette situation, l'article présente l'élaboration d'une intervention de groupe qui misait sur la création d'une coopérative de travail comme moyen de réinsertion collective.

Ce projet a été réalisé dans le cadre d'un stage de maîtrise en service social. Aussi s'agit-il de comprendre les résultats de l'expérience, autant sur le plan de l'atteinte des objectifs que du processus de groupe, dans les limites de ce contexte. Il demeure que la démarche ainsi que la perspective utilisées, en regard de différents courants théoriques sur lesquels l'intervention se fonde, méritaient d'être rapportées. 


\section{NOTE DE RECHERCHE}

\section{Vers le développement de l'autonomie économique des femmes: une expérience de groupe}

Pascaline COSSE

Étudiante à la maîtrise en service social Université de Montréal Intervenante psychosociale à la Clinique communautaire de Pointe-Saint-Charles, Montréal

S'il est une problématique sociale qui mérite d'attirer notre attention, c'est bien la situation de pauvreté ou de précarité économique vécue par beaucoup de femmes. Même si selon les dernières statistiques de 1993 les femmes sont de plus en plus nombreuses sur le marché du travail et qu'elles commencent à exercer les professions plus lucratives, leur rôle économique est encore jugé secondaire à celui de l'homme pour la majorité d'entre elles. Supportant souvent un stress psychologique et physique relié à la double tâche, puisque ce sont principalement elles qui donnent les soins aux enfants et aux aînés, sans reconnaissance pécuniaire, elles alternent périodes de travail et de chômage (Torjman, 1988; Bessette, 1987). À cause de ce temps 
consacré à la famille, l'écart sur le plan économique avec les hommes se creuse encore davantage. En 1991, les gains moyens des femmes occupant un emploi à plein temps étaient toujours de 30,5\% inférieurs à ceux des hommes (Statistique Canada, 1992). Épuisées, insuffisamment scolarisées et mal socialisées pour le marché du travail, beaucoup de femmes, surtout celles qui occupent des emplois précaires, décrochent de ce marché du travail inadapté à leur réalité de vie.

Les femmes issues des milieux défavorisés sont particulièrement vulnérables lorsqu'elles sont peu scolarisées ou qualifiées et qu'elles approchent le mitan de leur vie. Celles qui sont conscientes du risque élevé de pauvreté encouru pour le reste de leurs jours (Conseil national du bien-être social, 1990) cherchent à y échapper en optant pour la formule de la réinsertion socio-professionnelle. Le projet de réinsertion est souvent vécu individuellement, bien que des populations cibles telles que les jeunes et les femmes sont rejointes par des programmes d'aide particuliers.

Se regrouper pour partager des sentiments et échanger des trucs conduit à briser l'isolement, mais ne se révèle pas réellement une démarche de prise en charge collective. L'idée de se réunir entre femmes et de mettre en œuvre la solution consistant à créer une coopérative de travail par et pour elles-mêmes proposait la possibilité d'associer des énergies et de rendre complémentaires des habiletés propres à chacune.

L'intervention de groupe dont il est question dans le présent article relève d'une expérience-terrain qui s'est déroulée dans le cadre d'un stage de maîtrise et s'articule dans une démarche de recherche-action. Après avoir situé le contexte de l'intervention, les difficultés de réinsertion socio-professionnelle propres aux femmes et le groupe comme moyen pour une prise en charge collective, nous présenterons la méthode utilisée et les résultats de l'expérience. Enfin, nous analyserons les résultats autant en ce qui concerne le processus de groupe que l'atteinte des objectifs poursuivis.

\section{CONTEXTE ET DESCRIPTION DE L'INTERVENTION}

L'intervention de groupe que nous allons vous présenter a réuni dix femmes de la mi-novembre à la fin de mars 1992, pendant douze rencontres. L'implantation du projet de groupe a par ailleurs nécessité trois mois de préparation. Les objectifs poursuivis étaient les suivants: après avoir cherché et analysé les forces communautaires en présence, implanter un projet de groupe, pour et avec les femmes sans emploi, dans un milieu qui serait favorable à son développement. Ensuite, nous visions à amener le groupe à devenir porteur d'un projet de création d'une coopérative de travail à partir des acquis et des apprentissages réalisés en groupe. 
La démarche se situait dans une perspective de prise en charge collective et s'inspirait de deux orientations théoriques principales: le modèle à buts sociaux du service social de groupe (Weiner, 1961, 1966; Lewis, 1983) et le modèle autogéré (Mullender et Ward, 1991). Des principes de l'intervention féministe (Home, 1983) et aussi du courant de l'éducation des adultes (Kidd, 1971 ; Knowles, 1976) précisaient la nature du projet.

Enfin, le projet, parrainé par un organisme volontaire d'éducation populaire spécialisé dans le développement de coopératives de travail, a été réalisé dans le centre de femmes d'un quartier populaire dans le sud-ouest de Montréal. Une ouverture vers un partenariat local s'est opérée, puisqu'une corporation de développement économique et communautaire a apporté sa collaboration à la phase terminale de l'intervention en offrant de la formation afin que le groupe puisse poursuivre sa démarche.

\section{LES DIFFICULTÉS DE RÉINSERTION SOCIO-PROFESSIONNELLE DES FEMMES}

Les possibilités d'accès au marché du travail ne sont pas équivalentes pour les femmes et pour les hommes, pour les jeunes et pour les plus vieux. La place occupée sur le marché du travail apparaît être la principale source de disparité économique entre les sous-groupes de population mentionnés.

Paquette (1989) rapporte que « les différences qui apparaissent entre la situation socio-économique des hommes et des femmes s'expliquent à partir des choix scolaires féminins et masculins, des filières d'emploi spécifiques à chacun des sexes, du phénomène de la ségrégation professionnelle, du travail à temps partiel et du chômage ». Le chômage des femmes apparaît comme une problématique sociale importante. La durée du chômage féminin se révèle supérieure à celle du chômage des hommes au Québec (Paquette, 1989). La mise à pied et la perte d'emploi sont les principales raisons de situation de non-emploi. Mais, parmi les autres raisons avancées, on constate que seules les femmes invoquent le motif des obligations familiales, même si elles sont de moins en moins nombreuses à le faire. En 1987, 4,8\% des chômeuses avancent cette raison selon Paquette (1989).

Le rapport du Conseil national du bien-être social (1990) indique que le risque de se retrouver dans la pauvreté continue d'être beaucoup plus grand pour les femmes que pour les hommes. Les dix dernières années ont contribué à apporter des changements, que ce soit sur le plan législatif ou plus directement dans les programmes sociaux, autant au Canada qu'au Québec. Notons plus particulièrement les nouvelles dispositions en matière de discrimination en emploi, la modification de la loi sur le divorce et sur le partage des biens des époux, l'augmentation des prestations pour les familles à faible 
revenu. Pourtant, selon ce même rapport, les changements positifs survenus au cours de la dernière décennie ne se sont pas traduits par une diminution de pauvreté pour les femmes.

Devant l'augmentation du nombre de chômeurs et de chômeuses de longue durée et de personnes assistées sociales, la nécessité de développer des modes d'intervention pour faciliter l'insertion ou la réinsertion au marché du travail est apparue. Ces interventions s'articulent autour du concept d'employabilité. Cependant, le concept d'employabilité, selon Provost (1989), désigne « la gestion de l'exclusion du travail » et permettrait de la définir comme « relevant d'abord et avant tout de la responsabilité individuelle» (p. 73). L'auteure montre ainsi qu'en centrant l'explication du chômage sur les manques et les faiblesses des personnes, l'utilisation du concept d'employabilité nie les problèmes d'exclusion du travail et de chômage prolongé (p. 81).

Lorsqu'on regarde la problématique du chômage féminin sur le plan de l'employabilité, on néglige l'aspect structurel du phénomène. En effet, aujourd'hui, "l'espace et le temps consacrés au maternage et au travail domestique sont partagés, entremêlés et confrontés aux exigences du travail salarié » (Barrère-Maurisson, 1987; Corbeil et al., 1990: 101). Aussi les femmes, parce qu'elles sont mères ou qu'elles risquent de le devenir, devront combiner toutes sortes de stratégies pour concilier leurs rôles. Cela tient au fait que le milieu de travail est encore dominé par des valeurs masculines de rationalité, d'efficacité, de disponibilité et de compétition. Le fait d'être mère représente ainsi un handicap (Corbeil et al., 1990).

Les auteures expliquent comment les femmes contribuent alors à combler les emplois précaires (à la pige, sur appel, à domicile) et à temps partiel. Elles aboutissent aussi encore par épuisement au chômage. En effet, la tension liée au fait de concilier des rôles multiples conduit à un niveau élevé de stress physique et psychologique. Aussi l'alternance entre le travail salarié et le chômage peut-il être utilisé par les femmes comme un répit pour reconstruire leur état de santé (Bessette, 1987).

Le chômage des femmes doit être considéré comme un problème collectif et comme le reflet de l'inadaptation du marché du travail à leur réalité de vie. Il s'ensuit du même coup une mise en évidence du rapport entre leurs besoins en matière $d^{\prime}$ insertion/réinsertion et l'incohérence des réponses gouvernementales apportées par l'intermédiaire des programmes de développement de l'employabilité, si fleurissants ces dernières années.

Nous ne ferons pas ici l'inventaire des programmes de développement de l'employabilité ni une analyse approfondie de leurs méthodes d'intervention, parce que nous considérons que le problème est au départ mal posé. Les mesures de développement de l'employabilité accompagnent des politiques de soutien du revenu de moins en moins généreuses et vont dans le sens d'un 
appauvrissement, en particulier pour les femmes. La réforme de l'aide sociale (loi 37) a été dénoncée par maints groupes communautaires comme une humiliation à vivre au féminin. La récupération du travail effectué par les femmes, dans le cadre de programmes de développement de l'employabilité (ex.: distribution alimentaire dans les écoles à la suite de la mesure Pagé), était par ailleurs vivement dénoncée (Rose du Nord, 1991; Gmati et Robitaille, 1991). Ces mesures s'inscrivent bien dans ce courant conservateur actuel qui consiste à vouloir remettre les gens au travail comme si les chômeurs et chômeuses étaient responsables du chômage. Nous examinerons maintenant comment l'intervention de groupe dont il est question proposait d'envisager la réinsertion sous un angle différent.

\section{LE GROUPE COMME MOYEN DE PRISE EN CHARGE COLLECTIVE ET DE RÉINSERTION}

Les femmes, membres du groupe constitué, présentaient des points communs sur le plan de leurs conditions de vie. Un article récent (Cosse et Home, 1993) trace un portrait rapide de leur situation économique en précisant leur dépendance financière au conjoint ou à l'État. Les valeurs approuvées par le milieu préconisent l'importance d'être mère et valorisent les femmes dans ce rôle (Gingras, 1983). Ainsi toutes les femmes du groupe étaient mères à l'exception d'une et justifiaient en partie leur absence du marché du travail par cette raison. Veilleux (1991) mentionne que le fait de mettre au monde des enfants constitue un facteur d'appauvrissement des femmes. À la maternité, ajoutons le motif justifiant leur non-travail rémunéré. Contraintes d'occuper des emplois précaires n'offrant que des petits salaires et aussi soucieuses du bienêtre de leurs enfants, elles ont depuis de nombreuses années décroché du marché du travail.

Le rapport du Conseil canadien du bien-être social (1990) montre que lorsque le niveau de scolarité des femmes est bas, le retrait du marché du travail est envisagé. Veilleux (1991) parle ainsi de «travail confisqué » et voit le travail rémunéré des femmes s'inscrire dans un vaste système économique qui repose sur le travail non rémunéré de ces mêmes femmes. Elle ajoute que le conditionnement social incite les femmes à considérer leur activité professionnelle comme secondaire par rapport à leurs rôles conjugal et familial, donc à écourter leurs études ou à se diriger vers des emplois où elles pourront se consacrer en priorité à leur famille. Dans les milieux défavorisés, le chômage, l'aide sociale et le mariage sont les principales issues pour les filles qui choisissent d'abandonner leurs études. Cet engrenage se perpétue souvent d'une génération de femmes à l'autre (Chamberland, 1988). Les femmes justifient aussi leur retrait du marché du travail par les coûts des services de 
garde d'enfants qui risquent de trop gruger leurs faibles revenus. Nous voyons comment l'oppression des femmes est en partie reliée à leur manque de pouvoir sur le plan socio-économique.

\section{LE GROUPE COMME MOYEN D'ACCROÎTRE L'AUTONOMIE DES FEMMES}

Home (1991) stipule que la mobilisation des femmes vers un changement social peut être réalisée par le groupe. Aussi dans notre intervention, la pratique du groupe était vue comme un moyen d'opérer une prise en charge collective. On ne saurait trop insister sur l'importance du groupe comme moyen d'allier le changement personnel et le changement social, de diminuer I'isolement et la dépendance des femmes, tout en favorisant leur conscientisation et leur plus grande autonomie (Cardin et Home, 1983). Si le petit groupe est perçu comme la pierre angulaire du mouvement des femmes, les interventions qui s'y relient réussissent plus souvent le changement personnel que le changement social (Home, 1991). Les conditions socio-économiques des femmes comme facteurs déterminant leur maintien dans une situation $d^{\prime}$ impuissance et d'oppression sont ainsi peu souvent sujettes à intervention, peut-être parce qu'on ne sait pas précisément si la pauvreté des femmes est l'effet ou la cause de leur oppression.

De plus, les travailleuses et les travailleurs sociaux, qui souvent peuvent se sentir impuissants à susciter le changement social, décident d'éviter l'échec en se concentrant plutôt sur le changement personnel (Breton, 1991). Cependant, devant l'accroissement de la vulnérabilité des femmes, de leur risque de devenir pauvres et l'incapacité des programmes gouvernementaux à les intégrer de façon durable à la sphère économique autrement que par le moyen de l'assistanat, nous avons expérimenté une intervention de groupe visant la création d'emplois par l'intermédiaire du développement coopératif.

Limoges (1987) emploie le concept de chômage créateur et propose aux personnes privées d'emploi de redéfinir leur rapport au travail en entamant une démarche d'insertion différente, telle que la création de son propre emploi. L'idée rejoint ici les femmes dans leur capacité à opérer un transfert d'habiletés acquises dans le travail au foyer, le bénévolat ou encore au cours d'expériences de collaboration avec le conjoint ou un parent. Paré (1990) présente un outil d'intervention qui permet à des femmes de reconnaître ces acquis et de les redéfinir sous forme de compétences à considérer dans une perspective de travail salarié. En réunissant les femmes dans un groupe qui recherche une solution collective dans la création de leurs emplois, nous nous engagions dans une perspective de plus en plus courante ces 
dernières années, soit la création d'entreprises communautaires (Mathieu et Bourque, 1990). Devant la pénurie d'emplois, ces entreprises résultent d'un processus collectif de recherche de solutions par des chômeurs et des chômeuses dans des quartiers qui misent sur un développement économique et communautaire. En recherchant la complicité de plusieurs organismes du milieu, nous nous avancions dans une ouverture de la pratique du service social de groupe vers un travail de partenariat (Breton, 1991).

Fidèles à la tradition du service social des groupes, nous misions sur la participation des membres du groupe à la recherche de solutions au problème social (Lewis, 1983). Convaincues du potentiel des femmes, nous lancions le pari que le groupe pourrait devenir porteur d'un projet de coopérative de travail. Des expériences de coopératives gérées par des femmes existent (Descarreaux et Ouellette, 1986 ; St-Martin, 1990) et elles connaissent un réel essor depuis les années 80 (D'Amours, 1986). Elles sont cependant plus isolées dans les pays capitalistes que dans ceux en voie de développement où elles ont conduit à une certaine indépendance économique, à l'amélioration des conditions de vie et au changement social (Reynolds, 1989).

\section{SYNTHÈSE DE L'INTERVENTION}

L'intervention comportait douze rencontres sur une période de trois mois, précédée par quelques mois d'analyse des besoins et du milieu. Nous résumons ici l'essentiel de l'intervention décrit dans Cosse et Home (1993). On peut identifier trois étapes d'intervention. La première, l'organisation, visait à amener le groupe à se constituer et à démarrer. C'est à ce moment qu'il s'est trouvé un terrain d'appartenance (centre de femmes) et qu'il a établi ses normes et son contrat. À partir des modèles autogérés (Mullender et Ward, 1991), à buts sociaux (Lewis, 1983) et de l'intervention féministe, l'intervenante a joué essentiellement un rôle de médiatrice et de facilitatrice en insistant sur le partage d'autorité, la clarification des attentes et la mobilisation du leadership.

Après trois rencontres a débuté la phase d'information / formation, axée sur la formation du groupe au développement d'une coopérative de travail. Il y a eu définition des ressources tant à l'intérieur du groupe (compétences des membres) qu'à l'extérieur (ressources d'aide). En plus des modèles mentionnés ci-dessus, la travailleuse sociale a utilisé les principes de l'éducation des adultes pour lier les apprentissages visés aux expériences passées des membres. Elle a alors assumé le rôle d'enseignante en plus de ceux joués jusqu'à présent. La huitième rencontre a marqué le début de la phase de planification, qui visait la préparation à l'autonomie et la définition de créneaux d'activités économiques à partir des besoins de la communauté. 
La travailleuse sociale a mis l'accent sur la consolidation du leadership du groupe et le développement de liens avec les ressources du milieu (spécialisées dans l'élaboration de projets économiques). Malgré la diminution graduelle de son leadership, elle a dû parfois revenir à un rôle plus central, par exemple pour aider le groupe à résoudre des conflits. À la fin, elle a continué à jouer un rôle de personne-ressource en gardant un contact téléphonique (Home, 1983), afin de soutenir le groupe dans la poursuite de sa formation et son action.

\section{MÉTHODE D'ÉVALUATION ET INSTRUMENTS DE MESURE}

L'évaluation de cette intervention de groupe exige de prendre des précautions particulières quant au statut et au rôle empruntés par la chercheuse et aussi en ce qui concerne le choix des instruments de mesure. Notre fonction première était l'intervention. Les objectifs de celle-ci devaient passer avant ceux de la recherche. Cette dernière est cependant utile et nécessaire pour toute personne qui est intervenante-chercheure pour prendre un recul afin d'effectuer ainsi des ajustements susceptibles d'améliorer l'intervention (StArnaud, 1989). Notre recherche-terrain s'apparente à un projet pilote et, dans ce cas, il est indiqué d'utiliser plusieurs modèles de recherche, comme le mentionnent Darveau-Fournier et Home (1986). Nous y retrouvons donc trois types de recherche: la recherche évaluative, la recherche-action et la recherche féministe.

En ce qui a trait à la recherche évaluative, elle est de type formatif et porte à la fois sur l'appréciation des résultats et sur le processus d'intervention. La recherche-action met aussi l'accent sur le processus et pas seulement sur le produit (Stinson, 1978). On insiste alors sur l'exploration, l'expérimentation et la signification sociale du projet (Suchman, 1967). «L'expérience des femmes comme point de départ de la recherche, l'empathie, la collectivisation du problème, le statut de sujet et non d'objet pour les participantes ainsi qu'une relation égalitaire avec la chercheure et enfin l'ouverture vers la multidisciplinarité » sont des paramètres propres à la recherche féministe (Ouellet, 1991). Ils se retrouvent dans notre travail.

Les instruments de mesure sont au nombre de cinq. Nous avons principalement utilisé un cahier de bord, une grille d'observation des rencontres, une grille de développement d'un groupe (Dimock, 1970), remplie à quatre reprises. Un questionnaire d'accueil (rencontres pré-groupe avec les participantes) et une grille d'évaluation de chaque rencontre remplie par les membres individuellement nous ont aussi permis de déterminer les résultats de l'expérience, qui apparaissent être de deux types. Le premier touche I'atteinte des objectifs d'intervention (implantation du groupe et définition d'un projet de coopérative) et l'évolution du groupe. Le deuxième type de 
résultats traite du processus de groupe et des acquis en matière d'apprentissages tant collectifs qu'individuels.

\section{RÉSULTATS ET ANALYSE}

\section{Atteinte des objectifs}

Les premiers mois (septembre à fin novembre 1991) portaient sur l'implantation du projet de groupe. Heap (1987) considère que cette phase est particulièrement déterminante pour l'avenir d'un groupe. Aussi, au moment de cette expérience, le travail préparatoire visait-il à repérer les forces communautaires (valeurs et influences, réseaux de soutien et organismes d'aide à la création d'emplois) dans le milieu avant de recruter les femmes.

Il faut ouvrir la pratique du service social de groupe sur la communauté en développant un modèle de partenariat si nous voulons intervenir auprès des personnes difficiles à rejoindre, celles qui sont peu habituées à exercer un pouvoir et qui ont peur de prendre des risques (Breton, 1985, 1991). Les femmes vivant dans les quartiers populaires font partie de cette population surtout en ce qui touche le pouvoir économique. Le recrutement de membres pour mettre sur pied des groupes de changement social demande beaucoup d'énergie (Home, 1991 ; Lewis, 1983). Avant d'y procéder, il faut préalablement s'assurer de la présence des ressources matérielles et techniques dont on aura besoin plus tard.

Enfin, treize résidentes du même quartier dans le sud-ouest de Montréal ont été invitées à former le groupe. Elles fréquentaient pour la plupart le centre de femmes et la coordonnatrice connaissait celles qui étaient préoccupées par le désir de travailler de nouveau à l'extérieur du foyer. Le groupe ainsi constitué possédait une identité communautaire et avait un milieu d'appartenance naturel même si la travailleuse sociale aidée de la coordonnatrice était à l'origine du projet. Nous proposions à ces femmes de s'associer pour rechercher une solution à leurs besoins communs.

La présence d'un réseau de coopératives démontrait la croyance dans les principes de coopération et de solidarité dans le quartier. Enfin, la corporation de développement économique et communautaire et l'organisme de développement coopératif qui parrainait le stage s'annonçaient comme des partenaires pour établir le projet économique plus tard.

La réunion de dix membres, la disponibilité d'un local, d'un service de garderie, le repérage de partenaires possibles marquaient ainsi la réussite de I'implantation du projet après trois mois de préparation. II s'agissait alors de faire cheminer le groupe jusqu'à ce qu'il détermine une activité économique autour de laquelle les femmes pourraient démarrer leur entreprise. Selon l'éducation des adultes, ce créneau économique ne devait pas être n'importe 
lequel, mais exigeait d'opérer un transfert des forces de travail qu'elles se reconnaissaient à partir d'habiletés qu'elles avaient repérées et combinées les unes avec les autres. Ce créneau devait aussi répondre à leurs goûts.

Les participantes ont alors retenu deux projets: un service de rénovation de bicyclettes et celui d'un traiteur de repas. Le service de rénovation de bicyclettes visait la location / vente aux abords de la piste cyclable longeant le canal Lachine. L'idée de coudre des vêtements de sport était aussi retenue, puisque certaines d'entres elles avaient déjà travaillé dans une entreprise de confection de maillots de bain en lycra. La deuxième idée était de mettre en place un service de traiteur de repas emballés sous vide pour les organismes communautaires et les personnes seules habituées à un régime spécial. Plusieurs des membres, en particulier les femmes qui participent à des groupes d'achat et de cuisine collectifs, présentaient déjà des habiletés significatives pour ce genre d'activités.

La dernière phase du groupe a conduit les femmes à une prise de contact avec la responsable du développement des projets économiques locaux, qu'elles ont invitée au groupe. Par ailleurs, une représentante du groupe a rencontré le coordonnateur de la ressource en développement coopératif afin de lui présenter les résultats de la démarche du groupe et de connaître les possibilités d'aide offertes par l'organisme dans la poursuite du projet. Au terme de l'intervention, le groupe était composé de six femmes et cinq d'entres elles (la sixième étant enceinte) se sont inscrites à une formation sur l'élaboration de projets économiques de type coopératif offerte par un formateur spécialisé dans le domaine. En raison des limites de temps, l'intervention ne pouvait aller jusqu'à la mise sur pied de la coopérative de travail comme telle, la travailleuse sociale n'étant par ailleurs pas au fait de l'élaboration d'un plan d'affaire. L'intervenante a alors quitté le groupe, sachant qu'il restait encore beaucoup à faire, mais satisfaite de l'atteinte des objectifs de l'intervention sociale et admirative devant la persévérance et l'esprit créatif de ces femmes. Il s'agissait maintenant pour le groupe d'approfondir son projet sur le plan économique et de continuer à cheminer sur les bases qui avaient été posées dans les derniers mois de travail.

Nous savons qu'un processus d'appropriation (empowerment) ne se met réellement en place que lorsque les personnes peuvent agir sur les structures et sur les personnes qui détiennent le pouvoir dans l'environnement où elles se trouvent (Chamberland, 1988). Aussi faut-il se demander si le développement de l'autonomie économique des femmes, particulièrement de celles en milieu populaire, est suffisamment valorisé pour les soutenir dans de telles démarches. Nous abordons ce questionnement parce que nous savons aujourd'hui que I'intervention n'aura pas eu la portée espérée. En effet, le groupe s'est dispersé vers la fin de la formation à laquelle la majorité des participantes s'étaient inscrites. Des contacts téléphoniques entre les participantes et l'intervenante rapportent des éléments qui peuvent peut-être nous éclairer. 
Le groupe qui suivait la formation était constitué de deux sous-groupes, celui des femmes et un autre, composé d'hommes. Le formateur était lui aussi un homme. Dans l'autre groupe les participants avaient aussi un projet de développement coopératif mais, comme il s'agissait de distribution d'un produit, leur réseau de personnes participantes était insuffisant. Aussi ont-ils pensé à recruter de nouveaux membres parmi le groupe de femmes. Cette proposition a eu un effet de division au sein de celui-ci, plusieurs femmes abandonnant le projet collectif. Souvent, les femmes préfèrent se retirer plutôt que d'exprimer l'insatisfaction qu'elles ressentent (Home, 1986). Nous savons aussi qu'une des caractéristiques des personnes qui vivent de l'impuissance est d'exprimer leur exclusion du pouvoir en ne participant pas (Gingras, 1983; Breton, 1979).

Nous pensons que ce groupe de femmes ne pouvait supporter si tôt une participation dans une formation à composition mixte. Peu habituées à exercer du leadership, surtout lorsque celui-ci est insuffisamment reconnu, les femmes, parce qu'elles manquent de confiance en elles, perdent leur force dans les groupes mixtes (Home, 1991). Ce groupe ne pouvait compétitionner avec celui des hommes, à force égale, dans une formation de ce type, où l'on traite de notions économiques, juridiques et idéologiques. Les femmes sont souvent intimidées dans les groupes mixtes, elles ont tendance à se sousévaluer et à surévaluer les hommes, ce qui les rend vulnérables et faciles à manipuler (Home, 1983, 1991). Aujourd'hui, nous nous demandons s'il n'aurait pas été important d'éviter que le groupe de femmes ne suive ce type de formation dans un groupe mixte, afin qu'elles aient plus de temps pour affirmer leur style et les acquis réalisés jusqu'alors.

\section{Processus de groupe}

Les propos de Lewis (1983) illustrent une différence de nature dans les résultats lorsque celle-ci parle de «processus et de produit » dans la pratique du service social de groupe avec les groupes communautaires d'adultes.

Dans le processus de groupe, nous identifions des résultats sur trois plans: le développement du groupe en lui-même, les acquis obtenus sur le plan collectif et les apprentissages réalisés individuellement par les membres.

Les tableaux 1 et 2 organisent les résultats selon les trois phases de notre groupe, soit l'organisation, l'information/formation et la planification. Ces phases reprennent dans une certaine mesure l'appellation des phases de début, travail, transition et fin de Schwartz (1971). Nous les avons nommées ainsi pour mieux rendre compte de la variété des modèles utilisés. Ainsi I'organisation renvoie au modèle autogéré décrit par Mullender et Ward (1991), qui jugent important que ce soient les membres du groupe plutôt que les professionnels qui décident des moyens à utiliser pour atteindre le but. 
L'information/formation rappelle les méthodes de l'éducation des adultes, tandis que la planification renvoie au modèle à buts sociaux du service social et à celui des groupes de femmes qui s'inscrivent dans le changement social (Home, 1983, 1991).

Nous avons utilisé la grille de développement du groupe de Dimock (1970), afin de tracer l'évolution des cinq dimensions de celui-ci: le climat, l'engagement, l'interaction, la cohésion et la productivité. L'observation de quatre moments précis $\left(2^{\mathrm{e}}, 5^{\mathrm{e}}, 8^{\mathrm{e}}\right.$ et $11^{\mathrm{e}}$ rencontres) s'est faite par la coordonnatrice du centre de femmes plutôt que par l'intervenante. Celle-ci a rempli une grille comprenant treize questions. Chaque question comporte quatre niveaux que nous avons cotés sur une échelle de valeurs allant de 1 à 4 . Le tableau 2 dresse un bilan des acquis et des apprentissages importants réalisés au cours des différentes phases.

TABLEAU 1

Relevé de développement du groupe selon les phases

\begin{tabular}{l|ccc}
\hline \multirow{2}{*}{ Dimensions } & \multicolumn{3}{|c}{ Phases } \\
\cline { 2 - 4 } & Organisation & $\begin{array}{c}\text { Information/ } \\
\text { formation }\end{array}$ & Planification \\
\hline Climat & 3 & 4 & 4 \\
Engagement & 3,6 & 3,5 & 3,6 \\
Interaction & 2,6 & 3,2 & 3 \\
Cohésion & 2 & 2,5 & 2 \\
Productivité & 2,8 & 3,3 & 3,4 \\
Moyenne & $\mathbf{2 , 8}$ & $\mathbf{3 , 3}$ & $\mathbf{3 , 2}$ \\
\hline
\end{tabular}

Les données du tableau 1 indiquent que le groupe s'est développé au cours de l'intervention, mais que son évolution est plus marquée entre la première et la deuxième phase. Les exemples inscrits au tableau 2 illustrent ce développement au cours du processus d'intervention. Les acquis mentionnés attestent de l'atteinte du but que le groupe s'était fixé et retrace le cheminement du groupe au cours des trois phases. Sur le plan individuel, les apprentissages réalisés se sont traduits par l'acquisition d'habiletés techniques et de connaissances générales concernant l'entreprenariat coopératif ainsi qu'une plus grande maîtrise sur le plan socio-affectif. Nous allons discuter des résultats des deux tableaux en partant des cinq dimensions du tableau 1 et en détaillant par des exemples tirés du tableau 2, pour mieux rendre compte du processus. Le développement s'est surtout fait sur le plan du climat, de l'interaction et de la productivité, alors que l'engagement et la cohésion ont peu changé. 
TABLEAU 2

Acquis du groupe et apprentissage des membres selon les phases

\begin{tabular}{|c|c|c|}
\hline Phases & Acquis du groupe & $\begin{array}{c}\text { Apprentissage } \\
\text { des membres }\end{array}$ \\
\hline Organisation & $\begin{array}{l}\text { - Reconnaissance de } \\
\text { la situation commune } \\
\text { - Fermeture du groupe } \\
\text { - Structure de chaîne } \\
\text { téléphonique } \\
\text { - Rédaction d'un contrat } \\
\text { par les membres } \\
\text { du groupe } \\
\text { - Achat d'un cahier } \\
\text { de bord collectif }\end{array}$ & $\begin{array}{l}\text { - Sortir de son isolement } \\
\text { - Classer par ordre } \\
\text { de priorité ses besoins } \\
\text { personnels, familiaux } \\
\text { - Réorganiser } \\
\text { son emploi du temps } \\
\text { - Faire confiance } \\
\text { aux autres } \\
\text { - Prendre la parole } \\
\text { devant les autres } \\
\text { - Se conscientiser, } \\
\text { faire une analyse sociale } \\
\text { de sa situation }\end{array}$ \\
\hline Information/formation & $\begin{array}{l}\text { - Climat de confiance } \\
\text { entre les membres } \\
\text { - Sentiment de cohésion : } \\
\text { le groupe se donne } \\
\text { un nom } \\
\text { - Le groupe se perçoit } \\
\text { comme une association } \\
\text { - Début de prise } \\
\text { en charge collective }\end{array}$ & $\begin{array}{l}\text { - Modification } \\
\text { de l'image de soi } \\
\text { et des comportements } \\
\text { (perception de soi, de } \\
\text { son rôle dans la famille, } \\
\text { confiance en soi...) } \\
\text { - Développement } \\
\text { d'habiletés (animation, } \\
\text { leadership, rédaction, } \\
\text { nouveau vocabulaire, } \\
\text { résolution de conflits, } \\
\text { capacité d'analyse } \\
\text { et d'évaluation) }\end{array}$ \\
\hline Planification & $\begin{array}{l}\text { - Sélection de créneaux } \\
\text { - Invitation et visite aux } \\
\text { organismes-ressources } \\
\text { - Inscription en formation } \\
\text { - } \text { Transition, réengage- } \\
\text { ment du groupe } \\
\text { (rencontres hebdo- } \\
\text { madaires sans la t.s.) }\end{array}$ & $\begin{array}{l}\text { - Faire valoir ses idées } \\
\text { - Prendre des décisions } \\
\text { - Utiliser les ressources } \\
\text { du milieu } \\
\text { - Défendre et négocier } \\
\text { ses besoins } \\
\text { - Se projeter dans le futur }\end{array}$ \\
\hline
\end{tabular}

Le climat recouvre autant des dimensions physiques qu'émotives qui reflètent les conditions dans lesquelles le groupe a travaillé (Bédard, 1980). Le choix du centre de femmes comme lieu de rencontre et l'importance 
accordée dès le départ à l'échange et à l'expression des sentiments a permis l'établissement d'un climat détendu. Les acquis et apprentissages mentionnés corroborent ces données en indiquant des éléments qui révèlent la pertinence de la formule du groupe pour répondre aux besoins des membres. Les femmes ont rapidement utilisé le groupe pour exprimer et échanger sur leur situation de non-emploi. Elles ont ainsi pu mesurer que leur problème de réinsertion dépassait le cadre des difficultés personnelles. Dans la première phase, elles ont analysé «le pourquoi » de leur situation, une notion centrale dans le modèle autogéré (Mullender et Ward, 1991). Elles se sont rendu compte que le handicap majeur perçu par une personne (âge avancé) était différent de celui vécu par une autre (enfants en bas âge), mais que les deux constituaient tout autant un obstacle à une réinsertion réussie.

L'interaction a trait aussi au système de communication dans le groupe et désigne les rapports qui s'établissent entre les membres. Ces rapports étaient propices à la reconnaissance $d^{\prime}$ 'une situation commune. Le fait de pouvoir exprimer leurs inquiétudes a permis à plusieurs de sortir d'un isolement. De plus, l'emprunt d'une perspective féministe a rendu possible un rapprochement avec l'intervenante.

Il est intéressant de rapporter un événement qui s'est produit au cours de l'expérience. L'irrégularité de la participation de deux femmes rendait le groupe mal à l'aise et un manque de confiance se développait. Au moment d'une nouvelle absence des deux participantes, plusieurs membres exprimaient un début d'hostilité par rapport à cette situation. L'intervenante a alors encouragé le groupe à exprimer aux deux femmes leur manque de confiance. En nous servant ainsi de la technique féministe qui est de nommer le malaise vécu en partant des émotions que l'on ressent, nous avons pu identifier ce que Shulman (1979) appelle « un obstacle au travail ». La semaine suivante, les deux femmes avaient pu ainsi clarifier leur ambivalence à participer et signifiaient leur départ du groupe en exprimant leurs doutes et leurs insatisfactions.

Ce fait illustre bien une augmentation dans la capacité de définir un problème et de résoudre un conflit. Il témoigne de l'instauration d'un système de communication ouverte, souvent difficile dans les groupes de femmes qui ont été socialisées à retenir leurs sentiments négatifs (Home,1986).

La productivité d'un groupe se rapporte à la définition de son but, des objectifs qu'il se fixe et des moyens qu'il emprunte pour les atteindre (Dimock, 1970). La croissance de la productivité est observable aux deuxième et troisième phases. À la phase d'organisation, le groupe s'est employé à établir ses structures, ses normes et ses outils. Même si cela a pris un peu de temps, c'était nécessaire pour favoriser l'accroissement de l'autonomie du groupe (Lang, 1972). Avant de se centrer sur la tâche à la deuxième et à la troisième phase, il devait définir ses règles de fonctionnement. 
Comme la plupart des groupes féminins, il a adopté, dès le début, les principes de distribution du pouvoir à travers la prise de décision de manière collective et le partage du leadership (Home, 1991). L'achat d'un cahier de bord collectif illustre cette idée. En partageant ainsi le pouvoir, mais aussi les responsabilités, un ensemble d'apprentissages a été favorisé, comme la tenue du cahier et l'animation de la rencontre. En plus de ces habiletés techniques, il faut souligner ceux concernant davantage l'affirmation de soi alors que les femmes négociaient avec les ressources des horaires de formation à leur convenance ou des moyens de garde pour leurs enfants.

On note aussi une augmentation de la capacité d'analyse et d'évaluation par rapport à soi et aux autres alors que les femmes ont défini les habiletés de travail relatives aux expériences de travail au foyer, salarié ou bénévole. L'utilisation de l'outil «question de compétence» (COFFRE, Relais Femmes, ICEA, 1989), que nous avons adapté pour les besoins du groupe, nous a beaucoup aidés.

L'engagement dans un groupe est visible par la participation des membres et leur niveau d'appartenance (Bédard, 1980). Or, la participation au groupe a été stable dès les premières semaines et six membres sur dix sont allées jusqu'au bout. Trois femmes ont quitté le groupe parce qu'elles n'étaient plus intéressées, la quatrième a trouvé un emploi au cours de l'expérience. Selon Schwartz (1971), l'engagement des membres est nécessaire dans le processus de création de relations interpersonnelles, alors que Lewis (1983) y insiste pour favoriser «la réalisation du changement social».

Il faut relever l'effort qu'ont dû fournir certaines femmes en résistant aux pressions de la famille ou de l'entourage. En plus des rencontres de groupe les membres effectuent des lectures et remplissent des grilles d'évaluation chez elles afin de se concentrer sur leurs échanges de vues lorsqu'elles se rencontrent. Aussi, la fidélité au groupe est mise à l'épreuve par le milieu familial qui dans certains cas manifeste une réaction à la moindre disponibilité de la mère ou encore devant les nouvelles aspirations de la conjointe. Une femme rapportait que son mari se disait inquiet par rapport à son « retour d'impôts » qui risquait d'être atténué maintenant si sa femme se mettait à gagner de l'argent en poursuivant ce projet. Notons que deux femmes ont quitté le groupe sur les conseils de leur entourage. Home (1983) faisait remarquer que beaucoup de femmes sont trop opprimées ou trop écrasées pour posséder l'espérance nécessaire à toute entreprise d'action sociale.

Par ailleurs, il a été possible de reconnaître que la formule coopérative n'intéressait en fait pas toutes les membres. Nous avons contasté que certaines femmes avaient un tel besoin d'identification personnelle à combler qu'elles ne voulaient pas se retrouver à nouveau confondues dans une collectivité, où il faudrait encore composer avec les autres. L'objectif double des 
groupes de femmes est un avantage, mais aussi un inconvénient lorsqu'elles n'établissent pas toutes la même priorité en même temps (Home, 1991).

La cohésion s'exprime selon Dimock (1971) par l'unité, la solidarité, le sentiment du «nous ». Le fait de décider de la fermeture du groupe à la troisième rencontre indique un début de cohésion. Home (1986) mentionnait que le potentiel du développement de cohésion est moins grand dans les groupes ouverts constamment aux nouveaux membres. Nous remarquons qu'à la phase de l'information/formation, cette cohésion s'exprime par le fait que les femmes veulent donner un nom à leur groupe. L'appellation Arc-enciel sera choisie parce qu'elle met l'accent sur l'idée de progression dans les couleurs qui se fondent les unes dans les autres pour former un tout.

Par contre, la rencontre observée pour la dernière phase était marquée par l'inquiétude chez les membres à l'approche de la fin de l'intervention et donc du départ de l'intervenante. Aujourd'hui, nous pensons que la cohésion était insuffisante, ce qui a amené les membres à se diviser plutôt qu'à resserrer leurs liens au moment de la formation avec l'autre groupe. Comme le dit Lewis (1983), « un groupe de changement social doit être assez solide et cohésif pour résister aux pressions extérieures ». Il aurait fallu effectuer un suivi plus serré après l'intervention sociale pour veiller à la consolidation des acquis.

\section{CONCLUSION ET COMMENTAIRES}

L'intervention que nous avons analysée dans cet article avait pour objectif de trouver un milieu favorable à l'implantation d'un projet de groupe, réunissant des femmes sans emploi, afin de chercher des moyens pour retravailler. Elle visait à amener ce groupe à devenir porteur d'un projet de création d'une coopérative de travail. L'évaluation réalisée nous a permis de présenter des résultats positifs autant en ce qui concerne l'implantation du projet qu'en ce qui a trait à la définition de créneaux économiques par le groupe. Nous avons retracé aussi l'ensemble des acquis et des apprentissages qui ont pu être réalisés par le groupe et les membres. La force de notre intervention résidait dans la combinaison de méthodes empruntant les principes des théories du service social de groupe, de l'éducation des adultes et du féminisme.

Même si la portée de l'intervention n'a pas été celle escomptée et que les résultats ne sont pas généralisables, l'évaluation de cette expérience permet de penser que ces femmes, une fois réunies et orientées dans un cadre d'intervention spécifique, ont des capacités insoupçonnées pour trouver des solutions créatives à leurs difficultés communes en matière de réinsertion. Des obstacles persistent néanmoins dans la difficulté à pouvoir s'approprier des conditions favorisant leur propre développement. 
Chamberland (1988) mentionnait que l'expérience des personnes et leurs possibilités de produire des changements étaient reliées à leur possibilité d'exercer leur pouvoir dans des situations différentes. Certaines femmes, surtout celles que nous avons rencontrées au cours de cette expérience, sont tellement peu habituées à voir leur leadership valorisé qu'elles doutent qu'elles en possèdent un. II est vrai que les modèles de femmes entrepreneures qu'il nous est donné de voir abordent le profil d'une super-femme intellectuelle, à l'allure jeune et sexy.

Malgré l'atteinte de changements sociaux explicites, les groupes de femmes ne sont jamais inutiles par rapport à la prise de conscience et au développement de l'estime de soi (Home, 1991). Aussi pour en finir avec les cadres d'apprentissage traditionnels dominants, les femmes ne devraient-elles pas intervenir davantage sur les moyens qui leur sont offerts pour apprendre à réapprendre? (Butler et Wintram, 1991; Home, 1991)

\section{Références bibliographiques}

BARRÈRE-MAURISSON, M.A. (1987). «Structures économiques et structures familiales: émergence et construction d'une relation. La sociologie de la famille en question », L'année sociologique, 37 : 67-92.

BÉDARD, M. (1980). «Étude de l'évolution d'un groupe de personnes âgées », Service social, vol. 29, $\mathrm{n}^{\text {os }} 1$ et $2: 125-147$.

BESSETTE, L. (1987). "Chômage et dépression chez les femmes : le rôle du support social. Chômage et santé mentale, histoire et politique ", Revue de la santé mentale du Québec, vol. 12, n 2 : 82-99.

BOYER, M. et M.C. GUEDON (1989). "Chômage créateur: exploration à poursuivre ». Nouvelles pratiques sociales, vol. 2, $\mathrm{n}^{\circ} 2$ : 49-58.

BRETON, M. (1985). «Reaching and engaging people: Issues and Practice Principles », Social Work with Groups, vol. 8, no 3 : 7-21.

BRETON, M. (1991). "Ouvrir la pratique du service social de groupe sur la communauté: Vers un modèle de partenariat ». Texte inédit.

BRETON, M. (1979). "Nuturing abused and abusive mothers: the hairdressing group », Social Work with Groups, vol. 2, n 2 : 161-173.

BUtLer, S. et C. WINTRAM (1991). Feminist Groupwork, London: Sage.

CARDIN, M. et A. HOME (1983). "La pratique du service social avec les groupes de femmes ", Service social, vol. 32, $\mathrm{n}^{\text {os }} 1$ et $2: 170-185$.

Chamberland, C. (1988). "Les filles connaîtront-elles un jour l'expérience du pouvoir?", Revue canadienne de service social, vol. 5, été: 177-193.

COFFRE; RELAIS FEMMES; ICEA (1989). Question de compétence; Cahier de formation: un outil au service des femmes. Montréal : Coffre; Relais Femmes; ICEA.

CONSEIL NATIONAL DU BIEN-ÊTRE SOCIAL (1990). La femme et la pauvreté: dix ans plus tard. Rapport, Ottawa. 
Corbeil, C., F. Descarries, C. Gill et C. SeGuin (1990). « Des femmes, du travail et des enfants: des vies dédoublées », Nouvelles pratiques sociales, vol. 3, $\mathrm{n}^{\circ} 2$ : 99-115.

COSSE, P. et A. HOME (1993). «Groupes de femmes: outil de prise en charge collective », Actes du colloque Simone Paré de mai 1992. Sous presse.

D'AMOURS, M. (1986). "L'économie communautaire, casse-gueule ou possible? », La vie en rose: $21-24$.

DARVEAU-FOURNIER, L. et A. HOME (1986). «Un projet-pilote de soutien aux familles: aux confins de divers courants de recherche », Service social, vol. 35, $\mathrm{n}^{\text {os }} 1$ et $2: 126-140$.

Descarreaux, R. et D. Ouellette (1986). Le fonctionnement collectif dans des entreprises créées par des femmes. Coll. Essai, URECUS, Université de Sherbrooke.

DeVReuX, A.M. (1988). La double production. Les conditions de vie professionnelles des femmes enceintes. France, CSU.

DIMOCK, H.G. (1970). The Series on Leadership and Group Development. Factors in working with groups: How to analyze and evaluate group growth. Planning group development. Montréal : Université Sir George Williams.

GINGRAS, P. (1983). «Intervention auprès des femmes de classe populaire », Service social, vol. 32, $\mathrm{n}^{\text {os }} 1$ et $2: 89-100$.

GMATI, A. et J. ROBITAILLE (1991). "L'aide sociale et le cheap labor», Vie ouvrière, 233, 26-38.

HEAP, K. (1987). La pratique du travail social avec les groupes. Paris: ESF.

HOME, A. (1980). «Étude exploratoire de quatre types de changements survenus chez les membres de groupes féminins de conscientisation », Service social, vol. 29, $\mathrm{n}^{\text {os }} 1$ et $2: 158-181$.

HOME, A. (1983). "Les femmes et les groupes de changement social », Service social, vol. 32, nos 1 et $2: 50-76$.

HOME, A. (1986). «Les femmes, le sexisme et les groupes», les cahiers du GREMF (11). Québec: GREMF.

HOME, A. (1991). "Mobilizing Women's Strengths for Social Change: the Group Connection ", Social Work with Groups, vol. 14, nos 3 et $4: 153-173$.

KIDD, R. (1971). Adult education, the community and the animateur, dans Draper (ed.), Citizen Participation: Canada. Toronto: New Press, 137-158.

KNOWLES, H. (1976) The Modern Practice of Adult Education. New York: Association Press.

LAMOUREUX, H. (1989). Initiation à la vie coopérative. Guide de formation à I'intention des coopératrices et coopérateurs du travail. Comité provincial des coopératives de travail du Québec.

LANG, N. (1972). "A broad-range model of practice in the social work group », Social Services Review, vol. 46, n 1: 76-82.

LEWIS, E. (1983). «Le service social des groupes dans la vie communautaire», Service social, vol. 32 , $\mathrm{n}^{\text {os }} 1$ et $2: 32-49$.

LIMOGES, J. (1983). Chômage, mode d'emploi. Montréal : Éd. de l'Homme.

LIMOGES, J. (1987). "Prévention chômage. Chômage et santé mentale», Revue de la santé mentale du Québec, vol. 12, nº 2:106-113. 
MATHIEU, R. et R. BOURQUE (1990). "Les entreprises communautaires dans les services sociaux au Québec », Nouvelles pratiques sociales, vol. 3, $\mathrm{n}^{\circ} 2$ : 157171.

MUlLENDER, A. et D. WARD (1991). "Empowerment Through Social Action Group Work: The Self-Directed Approach », Social Work with Groups, vol. 14, n ${ }^{\text {os }} 3$ et $4: 125-139$.

OUELLET, F. (1991). "La recherche féministe», dans R. Mayer et F. Ouellet, F. (dir.), Méthodologie de recherche pour les intervenants sociaux. Boucherville: Gaëtan Morin éditeur, p. 205-232.

PAPELL, C. et B.H. ROTHMAN (1983). «Le modèle du courant central du service social des groupes en parallèle avec la psychothérapie et l'approche de groupe structuré », Service social, vol. 32, nos 1 et $2: 3-29$.

PAQUETTE, L. (1990). La situation socio-économique des femmes: faits et chiffres. Québec: Secrétariat à la condition féminine, Les Publications du Québec.

PARÉ, H. (1990). «Le travail au foyer et le travail bénévole des femmes sont porteurs de compétences», Nouvelles pratiques sociales, vol. 3, n 2 : 117-128.

Provost, M. (1989). "L'employabilité et la gestion de l'exclusion du travail », Nouvelles pratiques sociales, vol. 2, $\mathrm{n}^{\circ} 2: 71-82$.

REYNOLDS, S.F. (1989). "La création de revenus conduisant à l'indépendance, à des conditions améliorées et au changement social: Un cas concernant les femmes et le développement ", Éducation des adultes et développement, Association allemande pour l'éducation des adultes, vol. 32: 49-59.

Rose du Nord (1991). Les femmes et l'aide sociale. Enquête menée auprès de 140 femmes assistées sociales au Québec. Regroupement des femmes sans-emploi du nord du Québec.

SCHUMAN, E. (1967). Evaluative Research. New York: Russel-Sage.

SCHWARTZ, W. (1971). "L'utilisation des groupes en service social », dans W. Schwartz et S. Zalba (eds.), The Practice of Group Work. New York: Columbia University Press, 3-24. Traduction de Denise Taillefer.

SHULMAN, L. (1979). The skills of helping individuals and groups. Itasca, Ill. : Peacock. ST-ARNAUD, Y. (1989). "Praticien ou chercheur? » Sciences pastorales, vol. 8 : 63-79.

St-MARTin, N., D. Ouellette et J. Godbout (1990). L'autogestion au féminin: la création de coopératives de travail par des femmes, partie 1 : Revue de la littérature. Coll. Essai, URECUS, Université de Sherbrooke.

St-MARTin, N., D. Ouellette et J. Godbout (1990). L'autogestion au féminin: la création de coopératives de travail par des femmes, partie 2: Processus de création et besoins de formation. Coll. Essai, URECUS, Université de Sherbrooke.

StATISTIQUE CANADA (1992). Recensement 1989. Ottawa.

StINSON, A. (1978). Action Research for Social Action. Ottawa (occasional paper): Centre for Social Welfare Studies, Carleton University.

TORJMAN, R. (1988). L'écart des réalités : Combler l'écart entre les besoins des femmes et les programmes et services offerts. Document de référence. Ottawa: Conseil consultatif canadien sur la situation de la femme.

VEILLEUX, D. (1991). «Travailler mais rester pauvres, le sort collectif des femmes? L'autonomie financière: un défi », Femmes d'action, vol. 20, nº 5 : 14-17. 
WEIL, M. (1986). "Women, community and organizing», dans N. Van Den Berg et N. Cooper (eds.), Feminist visions for social work. Silver Spring, Maryland: NASW, p. 187-210.

WEINER, H.J. (1961). "Toward Techniques for Social Change», Social Work, vol. 6, $\mathrm{n}^{\circ} 2: 26-35$.

WEINER, H.J. (1964). «Social Change and Social Group Work Practice», Social Work, vol. 9, no 3 : 106-112. 